\title{
High energy gravitational scattering: a numerical study
}

\author{
Giuseppe Marchesini \\ Università di Milano-Bicocca, \\ Piazza della Scienza, 3, Milano 20126, Italy, and \\ INFN, Sezione di Milano-Bicocca, \\ Piazza della Scienza, 3 Milano 20126, Italy, and \\ Theory Division, CERN, Geneva, Switzerland \\ E-mail: Giuseppe.Marchesini@mib.infn.it
}

\section{Enrico Onofri}

\author{
Università di Parma, \\ via G.P. Usberti, 7 Parma 43100, Italy, and \\ INFN, Gruppo Collegato di Parma, \\ via G.P. Usberti, 7 Parma 43100, Italy \\ E-mail: Enrico.Onofri@unipr.it
}

ABSTRACT: The $S$-matrix in gravitational high energy scattering is computed from the region of large impact parameters $b$ down to the regime where classical gravitational collapse is expected to occur. By solving the equation of an effective action introduced by Amati, Ciafaloni and Veneziano we find that the perturbative expansion around the leading eikonal result diverges at a critical value signalling the onset of a new regime. We then discuss the main features of our explicitly unitary $S$-matrix down to the Schwarzschild's radius $R=2 G \sqrt{s}$, where it diverges at a critical value $b \simeq 2.25 R$ of the impact parameter. The nature of the singularity is studied with particular attention to the scaling behaviour of various observables at the transition. The numerical approach is validated by reproducing the known exact solution in the axially symmetric case to high accuracy.

KEywords: Spacetime Singularities, Models of Quantum Gravity, Classical Theories of Gravity. 


\section{Contents}

1. Introduction 1

2. The model 2

3. Solution by an iterative algorithm

3.1 Algorithmic details

3.2 The search for $R_{\text {crit }}$

4. Results 目

4.1 Critical behaviour 8

5. Conclusions 9

\section{Introduction}

We discuss in this paper the high-energy quantum string-gravity scattering by using the string formulation of Amati, Ciafaloni and Veneziano [1, 2]. Here we concentrate on the study of the high-energy elastic $S$-matrix for two colliding strings in the limit in which the string length $\lambda_{s}$ is negligible with respect both to the gravitational Schwarzschild radius $R$ and to the impact parameter $b$ of the the two colliding strings

$$
\frac{\lambda_{s}}{R} \rightarrow 0, \quad \frac{\lambda_{s}}{b} \rightarrow 0, \quad R=2 G \sqrt{s}, \quad \lambda_{s}=\sqrt{\alpha^{\prime} \hbar}
$$

where $\sqrt{s}$ is the center of mass energy. In this point-like-limit $d=4$ we rely on the observation that, because of the soft multi-loop strings amplitudes [1], the $S$-matrix exponentiates, in terms of an eikonal function of order $G s / \hbar$, times a function of $R^{2} / b^{2}$. In this limit the two colliding strings can be approximated by two massless particles

$$
S=e^{\frac{i}{\hbar} \mathcal{A}_{\mathrm{cl}}}, \quad \mathcal{A}_{\mathrm{cl}}=2 \pi G s \tilde{\mathcal{A}}_{\mathrm{cl}} .
$$

Here $\mathcal{A}_{\mathrm{cl}}$ is the classical value of an effective action given by a functional of gravitational fields constructed in terms of effective graviton vertices derived by Lipatov [3] in the study of the multi-Regge limit in (QCD and) gravity. The fields involved in the effective action $\mathcal{A}$ are the longitudinal and the transverse components of the metric tensor. The two colliding particles are described in terms of the energy-momentum tensor along the two light cone directions $x^{ \pm}=x^{0} \pm x^{3}$. 
The classical region to study is the case when $R$ is small and it approaches a critical value $R_{\text {crit }}$ at a fixed value of $b$. Here the critical value of $R$ is obtained by two approximations: we neglect rescattering terms and we neglect the infrared singular term of graviton interaction. Within these two approximations one is left with the critical behaviour

$$
R^{2} \frac{\partial}{\partial R^{2}} \tilde{\mathcal{A}}_{\mathrm{cl}}(b, s) \sim\left(1-R / R_{\text {crit }}\right)^{1 / 2} \Rightarrow \tilde{\mathcal{A}}_{\mathrm{cl}} \sim\left(1-R / R_{\text {crit }}\right)^{3 / 2}+\text { constant } .
$$

This critical behaviour has been derived in a modified model exhibiting axial symmetry (where the singular $\vec{b}$-dipole disappears) but here it will be reproduced in the actual case of a dipole at a distance $\vec{b}$.

It is amusing to note that our critical value for $R_{\text {crit }} / b \approx .455(1)$ is not far from the one obtained from the closed trapped surfaces in the axisymmetric case [4] signalling the transition from the present dispersive phase to the black-hole one, see also [5, 5].

The paper is organized as follows. First we introduce the model (section 2) and explain the two relevant approximations for the dipole picture with impact parameter $\vec{b}$. Then we explain the solution for $R \leq R_{\text {crit }}$ and discuss the search of the critical index (section 3 ). We then discuss the results and the behaviour of several observables near critical region with $R \leq R_{\text {crit }}$. Here we discuss also the region $R>R_{\text {crit }}$ in the axial case, which is not solvable by our iteration technique. We end with some conclusions.

\section{The model}

Here we recall the essential points of the effective action introduced in [1, 2] which are needed to describe our numerical study and we describe the two basic simplifications that are performed to give

$$
\begin{aligned}
\frac{\mathcal{A}}{2 \pi G s} & =\int d^{2} x\left(a_{+} t^{+}+a_{-} t^{-}-\frac{1}{2} \vec{\partial} a_{+} \cdot \vec{\partial} a_{-}+\frac{1}{2}(\pi R)^{2}\left(-\left(\vec{\partial}^{2} \phi\right)^{2}+2 \phi S_{a_{+} a_{-}}\right)\right) \\
S_{f, g}(\vec{x}) & \equiv \vec{\partial}^{2} f(\vec{x}) \vec{\partial}^{2} g(\vec{x})-\partial_{i} \partial_{j} f(\vec{x}) \partial_{i} \partial_{j} g(\vec{x})
\end{aligned}
$$

Here $a_{ \pm}(\vec{x})$ are the longitudinal graviton components (dotted lines in figure 1). Upon factorization the light-cone delta-function $\delta\left(x^{\mp}\right)$ respectively, they depend only on the transverse variables $\vec{x}=\left(x_{1}, x_{2}\right)$. In the same way $t_{ \pm}(\vec{x})$ correspond to the longitudinal components of the energy-momentum tensor associated to the two colliding particles at impact parameter $\vec{b}$

$$
t_{ \pm}(\vec{x})=\delta^{2}\left(\vec{x} \mp \frac{1}{2} \vec{b}\right) .
$$

The field $\phi(\vec{x})$ corresponds (upon factorizing the light-cone function $\theta\left(x^{+} x_{-}\right)$) to the transverse graviton component (the wavy line in figure 1a).

The first two terms of $\tilde{\mathcal{A}}$ correspond to the eikonal exchange of longitudinal gravitons (Coulomb scattering). This part of the action $(R=0)$ gives rise to the classical field

$$
a_{ \pm}^{(0) \mathrm{cl}}(\vec{x})=-\frac{1}{\pi} \ln \left(\lambda\left|\vec{x} \pm \frac{1}{2} \vec{b}\right|\right)
$$




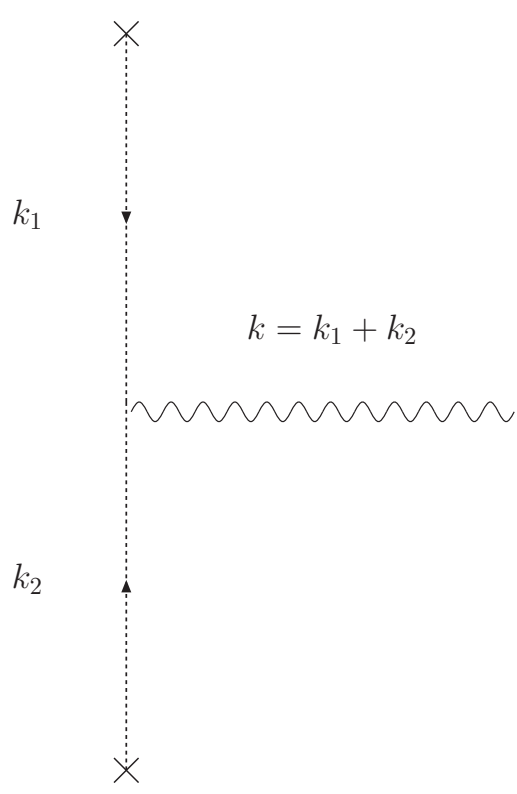

a)

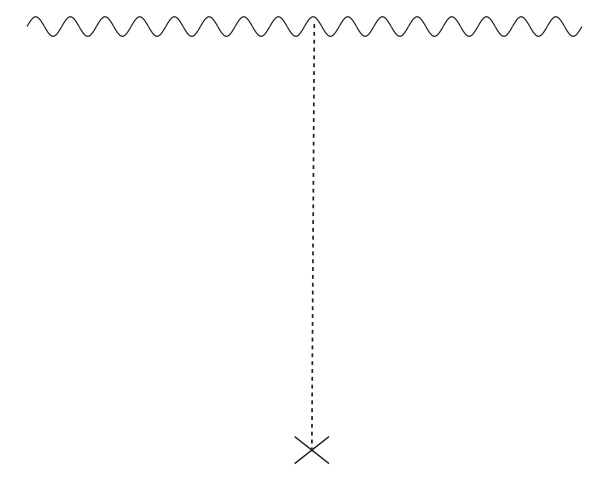

b)

Figure 1: (a) the scattering vertex between the two partons at distance $\vec{b}$. The crosses are the external particles. Dotted lines are $a_{ \pm}$and wiggly lines denote $\phi$. (b) The rescattering of partons.

with $\lambda$ an infrared cutoff. The corresponding classical action is given by

$$
\frac{\mathcal{A}^{(0) \mathrm{cl}}(b)}{2 \pi G s}=-\frac{1}{\pi} \ln (\lambda b),
$$

which shows that the infrared cutoff $\lambda$ is a divergent Coulomb phase contribution which does not affect observables. It reproduces Einstein's deflection angle $\sin \frac{1}{2} \theta_{\mathrm{cl}}=R / b$.

The other pieces of $\mathcal{A}$ correspond to quantum correction proportional to $R^{2} / b^{2}$. In particular the last term corresponds to the effective vertex [3] in figure 1a. The form of $\mathcal{A}$ in eq. (2.1) is based on two simplifications.

- No rescattering: the effective vertex in figure $1 \mathrm{~b}$, the rescattering term, is neglected. In this case the dependence of the fields on the light-cone directions $x^{+}$and $x^{-}$of the two colliding particles can be factored into the two delta functions $\delta\left(x^{ \pm}\right)$. Dropping this "trivial" dependence, one is left with the fields in the effective action which depend only on the two transverse space components $\vec{x}$.

- No infrared-singular transverse graviton component. The Lipatov vertex of figure 1a is given by $\left(\theta_{12}=\theta_{\overrightarrow{k_{1}} \overrightarrow{k_{2}}}\right)$

$$
V_{\mathrm{LLT}}^{\mu \nu}\left(\overrightarrow{k_{1}}, \overrightarrow{k_{2}}, \vec{k}\right) \sim \frac{1}{\vec{k}^{2}}\left(\sin ^{2} \theta_{12} \epsilon_{\mathrm{TT}}^{\mu \nu}(\vec{k})-\sin \theta_{12} \cos \theta_{12} \epsilon_{\mathrm{LT}}^{\mu \nu}(\vec{k})\right)
$$

with $\epsilon_{\mathrm{TT}}$ and $\epsilon_{\mathrm{LT}}$ the two independent transverse-traceless tensors of the graviton (see for example eq. 2.5 of ref. [2]). For $\vec{k} \rightarrow 0$ the first term $\sin ^{2} \theta_{12} / \vec{k}^{2}$ remains finite 
while the second one, $\sin \theta_{12} \cos \theta_{12} / \vec{k}^{2}$ diverges. In our study we neglect the $\epsilon_{\mathrm{LT}}$ transverse graviton component which brings in infrared divergences. The possible rôle of these singular components and the cancellation of the infrared singularities in the $S$-matrix has been discussed in 2] by using coherent states similar to what is done in QED and QCD [7].

In the space representation the first term of the vertex (2.5) gives rise to the $2 \phi S_{a_{+}, a_{-}}$ term in the action. Indeed

$$
F\left(\overrightarrow{k_{1}}\right) G\left(\overrightarrow{k_{2}}\right) \sin ^{2} \theta_{12} \quad \Rightarrow \quad S_{f, g}(\vec{x})
$$

with $f(\vec{x})$ and $g(\vec{x})$ the Fourier transforms of the two functions $F\left(\overrightarrow{k_{1}}\right)$ and $G\left(\overrightarrow{k_{2}}\right)$. The second term, infrared singular, gives $\left(\epsilon_{12}=\epsilon_{21}=-1\right)$

$$
F\left(\overrightarrow{k_{1}}\right) G\left(\overrightarrow{k_{2}}\right) \sin \theta_{12} \cos \theta_{12} \quad \Rightarrow \quad \vec{\partial} \partial_{i} f(\vec{x}) \cdot \vec{\partial} \partial_{j} g(\vec{x}) \epsilon_{i j}
$$

which for the free solution (2.3), with $f=a_{+}^{(0) c l}, g=a_{-}^{(0) c l}$, is proportional to $\vec{x} \wedge \vec{b}$. The contribution from this infrared singular vertex is neglected in eq. (2.1).

The equations of motion (2.1) are readily obtained by using the fact that $\phi S_{a_{+} a_{-}}=a_{ \pm} S_{\phi a_{\mp}}$ up to total derivatives. One has

$$
\begin{aligned}
\vec{\partial}^{2} a_{ \pm}(\vec{x}) & =-2 \delta^{2}\left(\vec{x} \pm \frac{1}{2} \vec{b}\right)+2(\pi R)^{2} S_{\phi a_{ \pm}}(\vec{x}) \\
\left(\vec{\partial}^{2}\right)^{2} \phi(\vec{x}) & =-S_{a_{+} a_{-}}(\vec{x})
\end{aligned}
$$

If $a_{ \pm}(x), \phi(x)$ is a solution, then

$$
\tilde{a}_{ \pm}(\vec{x})=a_{\mp}(\vec{b}-\vec{x}), \quad \tilde{\phi}(\vec{x})=\phi(\vec{b}-\vec{x}) .
$$

is also a solution. If the solution is unique (e.g. for small $R$ ) then it is symmetric. Perturbative corrections in $R^{2} / b^{2}$ to eq. (2.3) are finite. To first order the action is given by

$$
\frac{\mathcal{A}^{\mathrm{cl}}(b)}{2 \pi G s}=\frac{1}{\pi}\left(-\ln (\lambda b)+\frac{3 R^{2}}{8 b^{2}}+\cdots\right)
$$

Here one has used (2.1) thus neglecting the transverse $\epsilon_{\mathrm{LT}}$ graviton component. This latter has been computed in [2] and gives an additional contribution $R^{2} /\left(8 \pi b^{2}\right)$.

\section{Solution by an iterative algorithm}

In the small $R$ regime we solve eq. (2.8) by iteration $\left(\Delta=\vec{\partial}^{2}\right)$

$$
\begin{aligned}
a_{ \pm}(\vec{x}) & \leftarrow(1-\omega) a_{ \pm}(\vec{x})-\omega \Delta^{-1}\left(2 \delta^{2}(\vec{x} \mp \vec{b} / 2)-2(\pi R)^{2} S_{\phi, a_{ \pm}}\right) \\
\phi(\vec{x}) & \leftarrow(1-\omega) \phi(\vec{x})-\omega \Delta^{-2} S_{a_{+}, a_{-}}
\end{aligned}
$$


$\left(S_{f, g}\right.$ as given in eq. (2.1) $)$. Here $\omega$ is a "relaxation parameter" which may help convergence; in a linear context, the iteration converges if the r.h.s. has norm less than one, using the relaxation parameter can be useful if all eigenvalues have real part less than one. In our non-linear context the role of relaxation is not immediately clear. It is only used to check that divergence of the iteration is not changed by a trivial modification of the iteration mechanism. All results presented later on correspond to $\omega=1$.

In the actual implementation of the algorithm, we substitute the Dirac deltas with extended normalized Gaussian source terms

$$
\delta^{2}(\vec{x} \pm \vec{b} / 2) \rightarrow \mathcal{N} \exp \left\{\frac{-(\vec{x} \pm \vec{b} / 2)^{2}}{2 \sigma}\right\}
$$

since dealing with smooth functions improves the numerical stability; the Gaussian width $\sigma$ enters as a smearing parameter in the calculation.

\subsection{Algorithmic details}

The calculation is organized as follows. We choose a finite 2 -dimensional lattice of size $2 L$ and lattice spacing $2 L / N$, typically $L=64, N=256$; we introduce the dual lattice $\left(k_{1}, k_{2}\right)$ and define the basic differential operators in terms of $k_{i}$, i.e. $\Delta \rightarrow-k_{1}^{2}-k_{2}^{2}$, etc. The r.h.s. of the iteration map is then computed by going to Fourier space where necessary and taking local products of fields in coordinate space. This is done very efficiently for any $N$ by using FFTW [8], the best implementation of the fast Fourier transform to our knowledge. The main problem is to monitor the iteration and stop it when it can be decided that we have convergence or divergence. To monitor the iteration we choose a specific component of the action, namely the part proportional to $R^{2}(\mathcal{A}=2 \pi G s \tilde{\mathcal{A}})$

$$
\mathcal{M}=\frac{1}{2}(\pi R)^{2} \int d^{2} x\left(-\left(\vec{\partial}^{2} \phi\right)^{2}+2 \phi S_{a_{+} a_{-}}\right) \equiv \tilde{\mathcal{A}}_{\phi^{2}}+\tilde{\mathcal{A}}_{\phi a_{+} a_{-}}=R^{2} \frac{\partial}{\partial R^{2}} \tilde{\mathcal{A}} .
$$

A second monitoring device is provided by the fact, already used in [2], that on the solutions one has

$$
2 \tilde{\mathcal{A}}_{\phi^{2}}+\tilde{\mathcal{A}}_{\phi a_{+} a_{-}}=0 .
$$

When $\mathcal{M}$ shows a variation less that a certain prefixed value (say $10^{-6}$ ) then we stop the iteration and proceed to a more stringent convergence check based on the analysis of the linearized equation near the approximate solution. ${ }^{1}$ This is done as follows: let

$$
\left(\begin{array}{c}
a_{ \pm} \\
\phi
\end{array}\right) \leftarrow \mathcal{K}\left(a_{ \pm}, \phi\right)
$$

denote the iteration map; near convergence let $\phi=\tilde{\phi}+\delta \phi, a_{ \pm}=\tilde{a}_{ \pm}+\delta a_{ \pm}$where $\tilde{\phi}, \tilde{a}_{ \pm}$ represent the approximate solution obtained at the last iteration. Then we have

$$
\left(\begin{array}{c}
\delta a_{ \pm} \\
\delta \phi
\end{array}\right) \leftarrow\left(\begin{array}{ll}
\mathcal{D}_{11} & \mathcal{D}_{12} \\
\mathcal{D}_{21} & \mathcal{D}_{22}
\end{array}\right)\left(\begin{array}{c}
\delta a_{ \pm} \\
\delta \phi
\end{array}\right)
$$

\footnotetext{
${ }^{1}$ For $R>R_{\text {crit }}$ the iteration will diverge and the program will go in overflow unless we apply some strategy to early identify a divergent behaviour. A possible method is to monitor the curvature of the function $\mathcal{M}$ as a function of the iteration count.
} 
where the matrix $\mathcal{D}_{i j}\left(\tilde{a}_{ \pm}, \tilde{\phi}\right)$ is obtained by linearizing the equation and discarding quadratic terms in $\delta a_{ \pm}, \delta \phi$. The spectrum of $\mathcal{D}_{i j}$ is then computed. If the spectrum is contained in the unit circle then we conclude for convergence, otherwise for divergence. ${ }^{2}$ The linear operator $\mathcal{D}$ contains partial differential operators and the fields $a \pm, \phi$. Its largest eigenvalue, in absolute value, is computed ${ }^{3}$ by the Arnoldi algorithm contained in the mathematical library ARPACK [9].

\subsection{The search for $R_{\text {crit }}$}

Running the algorithm, we observe that the iteration converges at small values of $R$, where we effectively obtain the perturbative solution. However, increasing the parameter one finds that the algorithm ceases to converge at a value of $R=R_{\text {crit }}$ proportional to the impact parameter.

To identify the value of $R_{\text {crit }}$ where the transition occurs we adopt a "bisection method". Namely we start from an interval $\left[R_{\min }, R_{\max }\right]$ and set $R=\frac{1}{2}\left(R_{\min }+R_{\max }\right)$; if the iteration starting from $R$ is divergent we set $R_{\max }=R$ otherwise $R_{\min }=R$; the process is replicated until $R_{\max }-R_{\min }$ is less than a desired accuracy (typically $10^{-5}$ ).

The value thus obtained is however affected by the presence of "technical" parameters whose impact on the calculation must be carefully analyzed. The parameters are $L, N, \omega, \sigma$, i.e. infrared and ultraviolet cutoffs, the relaxation parameter and the width of the sources. On dimensional grounds one has

$$
R_{\text {crit }}=b F_{N}\left(\sigma / L^{2}, b / L, \omega\right),
$$

and, in principle, the limit $\sigma \rightarrow 0, L \rightarrow \infty, N \rightarrow \infty$ should be taken. It turns out that the iteration scheme is not sensitive to the relaxation parameter, hence we drop it from now on. On the other hand the dependence on infrared $(L)$ and ultraviolet $(\sigma)$ parameters is quite accentuated and some fitting procedure must be adopted in order to get the continuum infinite volume limit. Dependence on $N$ on the other hand is very weak above $N \sim 100$, as we argue in the next paragraph.

Continuum limit and finite size scaling. To estimate the $N$ dependence we performed a series of iterations starting with different parameters but keeping the adimensional ratios inside $F_{N}$ fixed. One finds that the results reach a flat plateau very soon (above $\mathrm{N}=100$ the numbers agree to 4 figures). This is surely due to the way differential operators are dealt with: any finite-difference scheme would introduce a systematic error of $O\left(N^{-n}\right)$ e.g. the roughest scheme corresponds to $\vec{\partial}^{2} \rightarrow 2 \sum_{i}\left(1-\cos \left(a k_{i}\right)\right) / a^{2} ;$ using $k^{2}$ itself in Fourier space makes the error exponentially small in $N$.

Having settled the $N$ dependence, systematic errors coming from $\sigma$ and $L$ should be addressed. A long run on a lattice of values for $b, \sigma$ and $L$ manifests a good scaling behaviour (i.e. the plot of $R^{2} / b^{2}$ is acceptably smooth, hence showing compatibility with a scaling behaviour).

\footnotetext{
${ }^{2}$ As an option we may consider the eigenvalue with largest real part: if this is larger than one we have divergence, otherwise convergence could be achieved by a suitable choice of relaxation parameter.

${ }^{3}$ Recent versions of Matlab ${ }^{\circledR}$ provide a user-friendly interface to the package.
} 


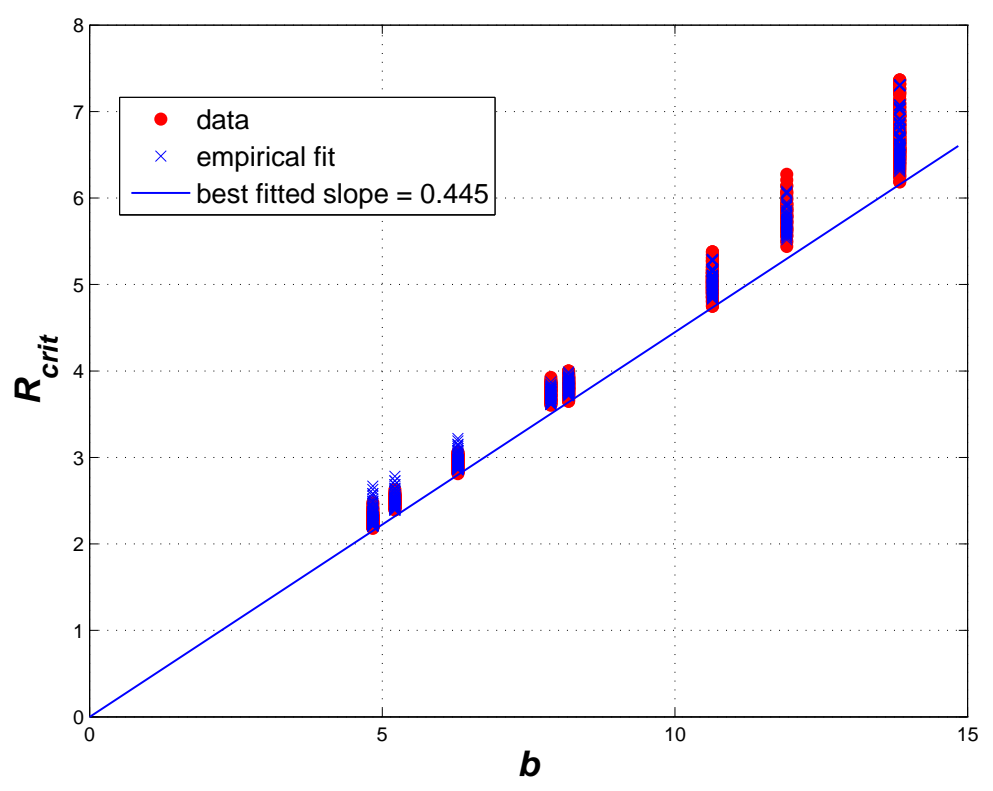

Figure 2: $R_{\text {crit }}$ vs. $b$ in the dipole model.

\section{Results}

The main result regards the dependence of the critical value of $R$ with respect to the impact parameter. We ran the program for a three-dimensional grid of values $\sigma \ll b \ll L)$ and we looked for a best fit to the $F_{N}$ in eq. (3.8) assuming a simple form

$$
F_{N}=\sqrt{1+\alpha_{1} \sigma / b^{2}+\alpha_{2}(b / L)^{2}+\alpha_{3}(b / L)^{4}+\ldots} .
$$

This gives (see figure 2)

$$
R_{\text {crit }} \approx 0.445(1) b .
$$

The evidence from the fit is that the correct slope corresponds to the envelope of the data from below, which can be interpreted in the sense that ultraviolet and infrared cutoffs both make the system more stable against collapse.

The axially symmetric case. A comparison with the result [2, 国 obtained in the case of axial symmetry, namely $R_{\text {crit }} / b=2^{1 / 2} / 3^{3 / 4} \approx 0.6204$ will support our analysis. In this case one of the sources is uniformly distributed around an annulus of radius $b$, while the other is localized at the center. The difference between this result and the value in eq. (4.2) suggests that the critical value, due to the non-linearity of the equations, may depend on the sources. In order to validate the accuracy of our numerical code, we applied it to the study of this axially symmetric case. A fit conducted with the same systematics as before gave the result

$$
R_{\text {crit }} \simeq 0.6216 b
$$

which presents the same pattern as in figure 2 and reproduces the exact slope to an accuracy of $0.5 \%$; this check makes us confident on the accuracy of our code and allows us to estimate the error in eq. (4.2) to less than $1 \%$. 


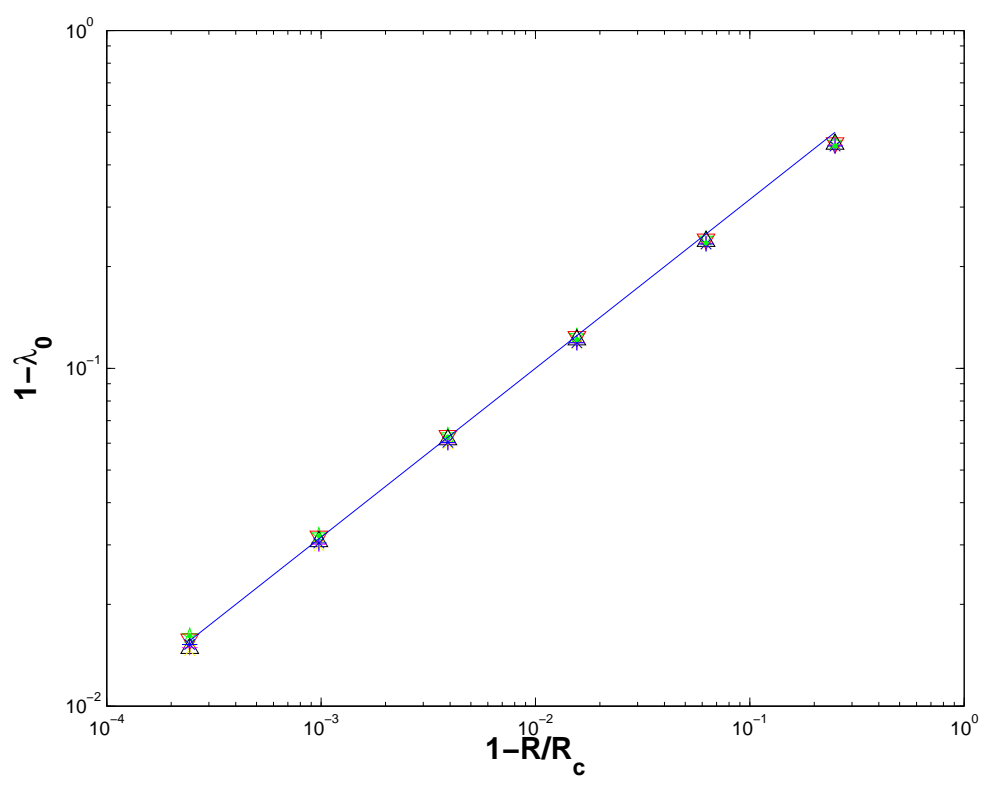

Figure 3: $\log -\log$ plot of the spectral radius as a function of $R$ in the dipole model.

\subsection{Critical behaviour}

In order to better understand the nature of the transition at $R=R_{\text {crit }}$ we shall now present some results about the critical behaviour of certain observables. The main fact we derive from our numerical data is that all observables that we examined have a scaling behaviour near the transition which can be reproduced very accurately by a square root singularity. This fact supports the conclusion that we are in presence of a genuine transition and not simply a breakdown of the iteration scheme. The argument is as follows: the iteration scheme represents an efficient way to sum up the perturbative expansion in the parameter $\mathcal{K}=2(\pi R)^{2}$; as such the iteration's convergence radius is regulated by the nearest singularity in the complex $\mathcal{K}$ plane. Our analysis shows that the divergence of the iteration scheme is caused by a singularity on the real line, which must then correspond to a physical singularity.

Spectral properties. Let's start with the spectrum of the linearized equation which is used in monitoring the convergence of the iteration algorithm. From the data a scaling property emerges which appears to be rather robust against variations of other parameters, namely the dependence of the spectral radius against $R$. Let $\lambda_{0}$ denote the spectral radius of the linearized equation: the plot of $1-\lambda_{0}$ as a function of $\sqrt{1-R / R_{\text {crit }}}$ is reported in the next picture (figure 3) and it suggests a relation of the type

$$
1-\lambda_{0}=C \sqrt{1-R / R_{\text {crit }}}
$$

with $C$ close to one.

The plot reports data from different values of $\boldsymbol{b}$ and it hints at the fact that there is universality in the relation $\lambda_{0}=\lambda_{0}\left(R / R_{\text {crit }}\right)$, i.e. the dependence on the impact parameter is only through the function $R_{\text {crit }}(b)$ and it is quite insensitive to the various cutoff parameters. 


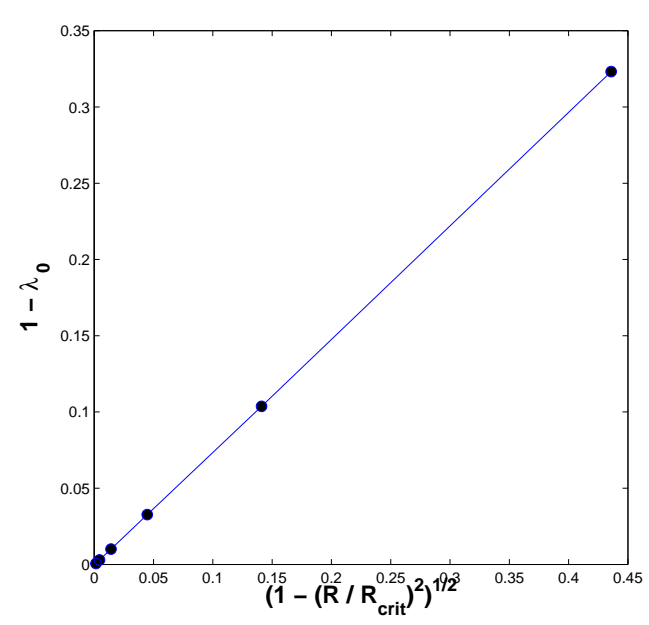

Figure 4: The scaling of the leading eigenvalue in the axial symmetric case below criticality

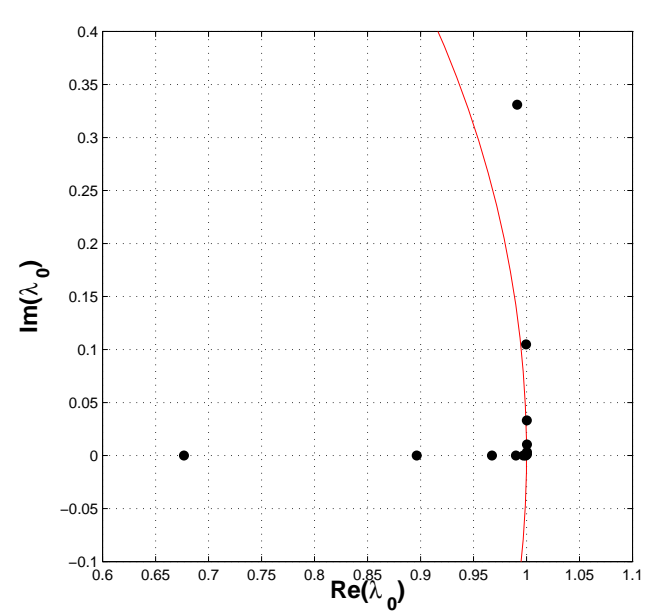

Figure 5: The leading eigenvalue above $R_{\text {crit }}$ in the axial symmetric case above criticality

A further investigation regards the region $R>R_{\text {crit }}$ in the case of axially symmetric sources. It is known [2, 团 that above the transition the solution becomes complex. We computed the spectrum of the linearized equation around the exact solution. The spectral radius is compatible with the analytic continuation of eq. (4.4), namely the largest eigenvalue gets an imaginary part — see figure 0 .

Classical Action, $h_{T T}$ and $h_{L T}$. Near criticality we examined other observables, namely global quantities like

- the action piece $\mathcal{M}$ as in eq. (3.4);

- $\int d^{2} x h_{\mathrm{LT}}^{2}$, which does not enter the action in the present approximation;

- $h_{\mathrm{TT}}=\Delta \phi$, integrated over a finite region $\propto b$.

As expected, we observed the same square root behaviour for $\mathcal{M}$

$$
\mathcal{M}=R \frac{\partial}{\partial R} \tilde{\mathcal{A}}(b, R) \sim \sqrt{1-R / R_{\text {crit }}}
$$

and for other cases - see figure 6. By integrating over $R$, one gets eq. (1.3).

\section{Conclusions}

We have neglected the string-size effects for $R, b \gg \lambda_{s}$ (with $\lambda_{s}$ the string effect) and studied the region without rescattering and without trace infrared effects for $R$ small compared to $b$ in (2.1). We arrived at the critical region in (4.5) with the square root behaviour ensured for various decades. This result nicely agrees with that obtained in the axially symmetric case [2, 汪. The fact that at the transition an eigenvalue 1 appears in the spectrum of the linearized equation shows that the solution (if any) at $R>R_{\text {crit }}$ is unstable against 

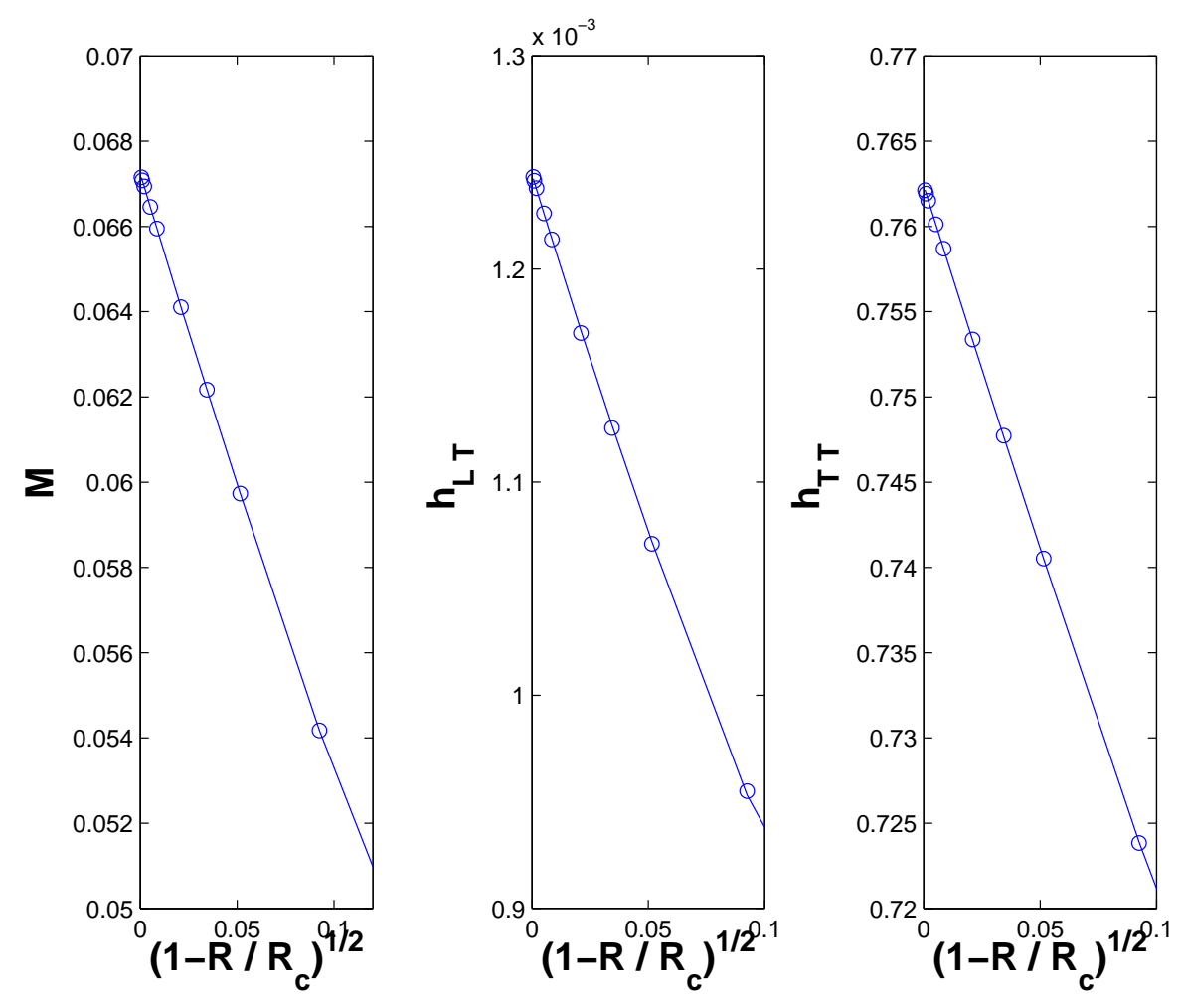

Figure 6: Scaling behaviour of $\mathcal{M}, h_{T T}$ and $h_{L T}$ near Local observables.

small perturbations, hence features that have been considered irrelevant in the derivation of eq. 2.1 may come back as relevant.

Finally, it is interesting that the critical value of $b_{\text {crit }} \approx 4.5 G \sqrt{s}$ that we find is not far from the values 4 obtained in similar studies of closed trapped surfaces which discriminate the transition from the present dispersive phase to the black-hole one. It is interesting that, taken at face value, our result would increase the cross section for black hole production given in [5], but we were unable to find a physical justification for this fact.

\section{Acknowledgments}

We wish to thank Gabriele Veneziano and Jacek Wosiek for valuable discussions.

\section{References}

[1] D. Amati, M. Ciafaloni and G. Veneziano, Effective action and all order gravitational eikonal at planckian energies, Nucl. Phys. B 403 (1993) 707.

[2] D. Amati, M. Ciafaloni and G. Veneziano, Towards an S-matrix description of gravitational collapse, JHEP 02 (2008) 049 arXiv:0712.1209.

[3] L.N. Lipatov, High-energy scattering in QCD and in quantum gravity and two-dimensional field theories, Nucl. Phys. B 365 (1991) 314. 
[4] G. Veneziano and J. Wosiek, Exploring an S-matrix for gravitational collapse, arXiv:0804.3321; Exploring an S-matrix for gravitational collapse II: a momentum space analysis, arXiv:0805.2973.

[5] D.M. Eardley and S.B. Giddings, Classical black hole production in high-energy collisions, Phys. Rev. D 66 (2002) 044011 gr-qc/0201034;

S.B. Giddings and V.S. Rychkov, Black holes from colliding wavepackets, Phys. Rev. D 70 (2004) 104026 hep-th/0409131.

[6] E. Kohlprath and G. Veneziano, Black holes from high-energy beam-beam collisions, JHEP 06 (2002) 057 gr-qc/0203093.

[7] P.P. Kulish and L.D. Faddeev, Asymptotic conditions and infrared divergences in quantum electrodynamics, Theor. Math. Phys. 4 (1970) 745 Teor. Mat. Fiz. 4 (1970) 153;

S. Catani, M. Ciafaloni and G. Marchesini, Noncancelling infrared divergences in $Q C D$ coherent state, Nucl. Phys. B 264 (1986) 588.

[8] M. Frigo and S.G. Johnson The design and implementation of FFTW3, Proceedings of the IEEE 93 (2005) 216.

[9] R.B. Lehoucq, D.C. Sorensen and C. Young, ARPACK users' guide, SIAM, Philadelphia (1998); MATLAB's implementation: R. Radke's thesis, online at http://www.caam.rice.edu/software/ARPACK/DOCS/radke.ps.gz. 